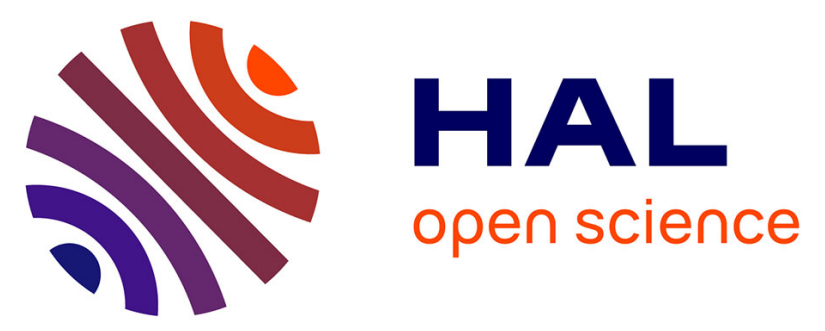

\title{
Capacitive Energy Conversion With Circuits Implementing a Rectangular Charge-Voltage Cycle-Part 1: Analysis of the Electrical Domain
}

Dimitri Galayko, Andrii Dudka, Armine Karami, Eoghan O'Riordan, Elena Blokhina, Orla Feely, Philippe Basset

\section{To cite this version:}

Dimitri Galayko, Andrii Dudka, Armine Karami, Eoghan O'Riordan, Elena Blokhina, et al.. Capacitive Energy Conversion With Circuits Implementing a Rectangular Charge-Voltage Cycle-Part 1: Analysis of the Electrical Domain. IEEE Transactions on Circuits and Systems I: Regular Papers, 2015, 62 (11), pp.2652-2663. 10.1109/TCSI.2015.2451911 . hal-01240996

\section{HAL Id: hal-01240996 \\ https://hal.science/hal-01240996}

Submitted on 16 Dec 2015

HAL is a multi-disciplinary open access archive for the deposit and dissemination of scientific research documents, whether they are published or not. The documents may come from teaching and research institutions in France or abroad, or from public or private research centers.
L'archive ouverte pluridisciplinaire HAL, est destinée au dépôt et à la diffusion de documents scientifiques de niveau recherche, publiés ou non, émanant des établissements d'enseignement et de recherche français ou étrangers, des laboratoires publics ou privés. 


\title{
Capacitive Energy Conversion With Circuits Implementing a Rectangular Charge-Voltage Cycle-Part 1: Analysis of the Electrical Domain
}

\author{
Dimitri Galayko, Member, IEEE, Andrii Dudka, Armine Karami, Student Member, IEEE, \\ Eoghan O'Riordan, Student Member, IEEE, Elena Blokhina, Senior Member, IEEE, Orla Feely, Fellow, IEEE, \\ and Philippe Basset
}

\begin{abstract}
Capacitive kinetic energy harvesters (KEH) employ conditioning circuits which achieve a dynamic biasing of the transducer's variable capacitor. This paper, composed of two articles Part 1 and Part 2, proposes a unified theory describing electrical and electromechanical properties of an important and wide class of conditioning circuits: those implementing a rectangular chargevoltage cycle. The article Part 1 introduces a basic configuration of conditioning circuit implementing an ideal rectangular $Q V$ cycle, and discusses its known practical implementations: the Roundy charge pump with different flyback mechanisms, and configurations based on the Bennet's doubler. In Part 1, the analysis is done in the electrical domain, without accounting for electromechanical coupling, while in Part 2, the full electromechanical system is analyzed. An optimization approach common to all configurations is proposed. A comparison is made between different topologies and operation modes, based on the maximal energy converted in one cycle under similar electrical and mechanical conditions. The last section discusses practical implementation of circuits with smart and adaptive behavior, and presents experimental results obtained with state-of-the art MEMS capacitive KEH devices.
\end{abstract}

Index Terms-Capacitance, circuit analysis, DC-DC power conversion, energy harvesting, power systems, switched systems.

\section{INTRODUCTION}

M ICROSCALE kinetic energy harvesters (KEH) are considered a useful complement to other available power sources such as chemical batteries and solar cells. Microscale KEHs are a relatively new class of device, which emerged a little over a decade ago [1]. The interest in microscale energy harvesting is explained by the success of micromachining technologies allowing a fabrication of very efficient miniature electromechanical systems (MEMS). KEHs are complex heterogeneous systems: they require advanced engineering of mechanical and electrical parts, and their operation is described

Manuscript received February 24, 2015; revised May 11, 2015; accepted June 08, 2015. Date of publication August 20, 2015; date of current version October 26, 2015. This paper was recommended by Associate Editor K.-H. Chen.

D. Galayko, A. Dudka, and A. Karami are with Sorbonne Universités, F-75005 Paris, France (e-mail: dimitri.galayko@lip6.fr; andrii.dudka@lip6.fr; armine.karami@gmail.com).

E. O'Riordan, E. Blokhina, and O. Feely are with School of Electrical, Electronic and Communications Engineering, University College Dublin, Ireland (e-mail: eoghan.o-riordan@ucdconnect.ie; elena.blokhina@ucd.ie; orla.feely@ucd.ie).

P. Basset is with Université Paris-Est, ESYCOM, ESIEE Paris, 93162 Noisy Le Grand, France (e-mail: p.basset@esiee.fr).

Color versions of one or more of the figures in this paper are available online at http://ieeexplore.ieee.org.

Digital Object Identifier 10.1109/TCSI.2015.2451911

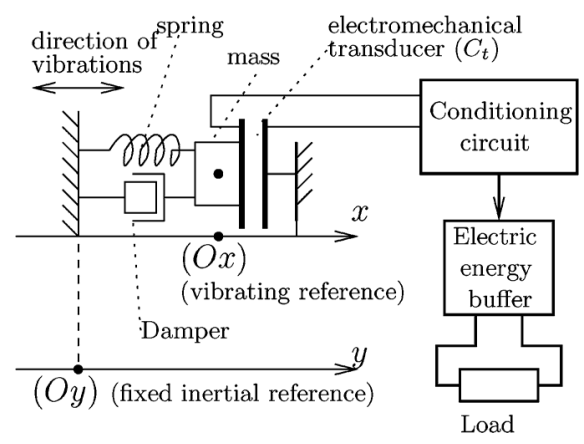

Fig. 1. Structural view of a kinetic energy harvester with capacitive interface.

by nonlinear multiphysics models. The electromechanical conversion mechanism is the main aspect distinguishing different implementations of KEH.

The diagram in Fig. 1 presents the structure of a KEH placed in a vibrating reference frame $O x$, which vibrates with an acceleration $a_{\text {ext }}(t)$ with regard to an inertial reference frame $O y$. A mobile mass, when considered in the frame $O x$, is subjected to a force equal to $-m a_{e x t}(t)$, where $m$ is the value of the mass. This force is a source of mechanical energy. The mass may be freely moving [2] or may be coupled with the vibrating frame $O x$ by a spring, as shown in Fig. 1 .

Electromechanical transducers have two mechanical terminals: one is fixed to the vibrating frame, and the other is attached to the mobile mass. When the mobile mass moves, the electricity is generated at the electrical terminals of the transducer. The physics of this process depends on the transducer implementation. For any transduction mechanism, the KEH requires a power management system which transforms the harvested electricity into a form suitable for the load supply (e.g., rectification, DC voltage stabilization, etc.). Indeed, the output voltage may only be a few hundreds of millivolts in the case of electromagnetic or piezoelectric harvesters, and special start-up and voltage level up circuits are necessary. On the contrary, capacitive transducers (as in Fig. 1) may generate tens of volts, and downward DC-DC conversion is needed. The electronics interfacing with the energy harvesters are usually designed under severe restrictions due to the power consumption, and are often integrated on silicon for the sake of miniaturisation [3], [4].

Unlike piezoelectric and electromagnetic transducers, capacitive transducers are passive devices. In addition to the above mentioned interface with the load and the power management, they require a special electrical conditioning (biasing) in order 


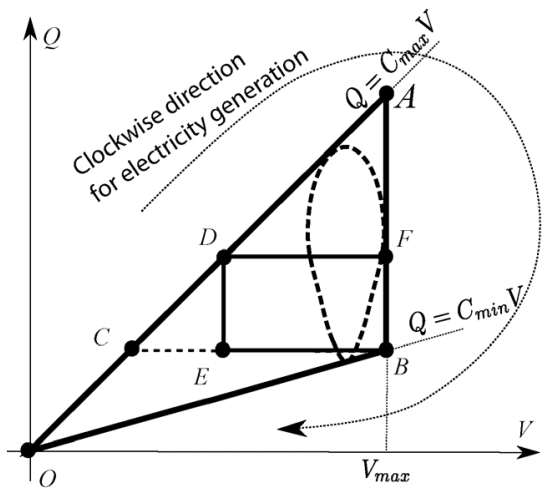

Fig. 2. Diagram showing all charge-voltage cycles which have been used to date for conditioning of capacitve transducers in KEHs: OCB (constant-charge), OAB (constant-voltage), DFBE (rectangular) and "tear drop" cycle corresponding to the continuous conditioning circuit or KEH with electret layer [5]. All cycles are drawn for the same extreme values of the variable capacitance $\left(C_{\min }, C_{\max }\right)$ and for the same maximal voltage $V_{\max }$

to convert motion into electricity. The implementation of efficient conditioning circuits for electrostatic $\mathrm{KEH}$ is hindered by a low power level available from the electrostatic conversion, hardly more that tens of microwatts per $\mathrm{cm}^{2}$.

In capacitive transducers, the mechanical energy is converted into electricity when the transducer is biased while its capacitance decreases, i.e., when electrical force does negative mechanical work on the mass. The electromechanical energy conversion process is characterized by the charge-voltage diagram (said "QV diagram"), which is a graphical representation of the evolution of the electrical state of a capacitive transducer. For a periodic variation of the transducer capacitance, the QV diagram is a loop whose area is numerically equal to the energy converted during a cycle. Moreover, in relation to the energy conversion process, the shape of the QV diagram is an obvious basis for the classification of conditioning circuits. Fig. 2 presents all QV diagrams implemented by known conditioning circuits.

One of the main roles of a conditioning circuit is to provide the transducer with a dynamic voltage biasing corresponding to the desired QV cycle. If the maximum voltage supported by the used transistor technology is limited to $V_{\max }$, the dynamic biasing allowing the most optimal electromechanical conversion is achieved by a circuit called "constant voltage circuit" [6] (cycle OAB in Fig. 2). However, since the early noughties, several architectures of conditioning circuits for capacitive transducers have been proposed: the continuous conditioning circuit [7] (tear drop QV cycle, cf. Fig. 2), circuits implementing the constant charge QV cycle [6] (triangular QV cycle OCB), circuits based on a charge pump [8] and recently circuits based on Bennet's doubler (rectangular QV cycle DFBE). Such a large number of concurrent solutions is explained by the difficulty in the practical implementation of the conditioning circuit with the constant voltage operation. The evolution of the conditioning circuits goes toward the simplification of the external management required for their operation: while the constant voltage and constant charge circuits require two synchronized switches at each period of vibration, the charge pump based circuit requires only one switching over several periods of vibrations. The Bennet's doubler based circuit does not require controlled switching at all.
Conditioning circuits based on the charge pump have been considered since 2002 (cf. basic architecture by Roundy et al. [8]); their great advantage is an automatic synchronisation of the charge flow on the capacitive transducer with the mobile mass vibration. Their common property is the rectangular shape of the QV diagram. The basic charge pump is usually complemented with a flyback, which defines its optimal steady-state operation (cf. Section III-A and III-B).

Recently, a new topology of conditioning circuit whose architecture is inspired by the Bennet's electricity doubler has been discovered. In comparison with previous conditioning circuits, these circuits increase their initial biasing by using the energy converted from the mechanical domain without employing any inductive $D C$-DC conversion. It was recently shown that these circuits also implement a rectangular QV cycle, and by consequence, they should be considered as belonging to the same family as the circuits based on the charge pump.

To date, conditioning circuits based on Roundy's charge pump and on the Bennet's doubler have been analyzed separately, and only from the point of view of electrical engineering. The published works have focused on experimental demonstration of the energy conversion with these circuits. However, a fundamental study of the behavior of these circuits as a part of an electromechanical system such as a KEH is still missing. An analysis of electromechanical behavior due to the transducer has never been done; it is not known how a transducer which operates following a rectangular QV cycle impacts the mechanical domain of the harvester. Understanding the common properties of these two apparently different architectures, but having very similar QV diagrams, must help in the optimization of converted power for given design specifications.

This paper, composed of two articles Part 1 and Part 2, presents a complete electrical and electromechanical analysis of a KEH which uses a capacitive transducer biased by a rectangular QV cycle. This analysis will be based on tools proposed for nonlinear KEH in previous works [9] allowing a nonlinear analysis of coupled electromechanical systems.

Part 1 (this article) addresses the analysis of the conditioning circuits from the electrical point of view. It is organized as follows. In Section II, we present properties and main figures of merit for an energy harvester employing a rectangular QV cycle. We explain how an ideal conditioning circuit implementing a QV cycle may be optimized from the electrical point of view, as a function of different system-level limitations. In Section III we address the practical implementation of a rectangular QV cycle. The geometric parameters of the cycle will be related to the physical parameters of the conditioning circuits based on the Roundy charge pump and Bennet's doubler. We explain how the circuits should be optimized so as to maximize the power of the electricity generation for different practical hypotheses, and we will highlight differences between these two circuits. We present the concept of adaptive operation of the circuits based on the Roundy's charge pump, and show how smart electronics may ensure that for any change in the external vibrations, the operation of the circuit is still optimal. Section V discusses practical implementations and experimental results.

Part 2 of the paper is devoted to the analysis of the system " conditioning circuit; resonator; transducer\}." A precise analytical model is developed for the Roundy charge pump with resistive flyback. This model is then solved with the multiple 


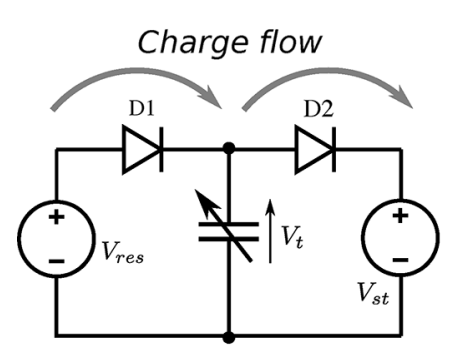

a)

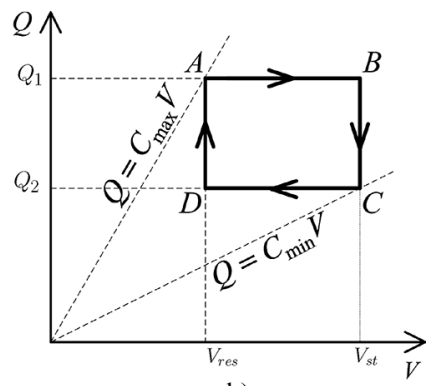

b)
Fig. 3. Schematic of an ideal conditioning circuit implementing a rectangular charge voltage cycle (a) and the plot of the corresponding QV cycle (b).

scales method, which belongs to the family of the perturbation techniques. The method predicts the dynamics of the mobile mass vibration, resulting from the action of the external forces (vibrations), from mechanical properties of the resonator, and from the action of the transducer biased by the conditioning circuit. The knowledge about the resonator amplitude provides information about the dynamic of the transducer capacitance variation. Hence, with the electrical analysis presented in Part 1, it is possible to know the rate of the energy conversion (the power). Predictions on the system dynamics resulting the proposed analysis method agree very well with the experiment, done with a fabricated MEMS device.

\section{A PRimitive CiRCuit ImPlementing A ReCtANGUlaR QV CYCLE}

Since in Part 1 only the electrical behavior is considered, in this article the resonator and transducer are modeled as a variable capacitor. Its capacitance $C_{t}(t)$ varies cyclically in time between its extreme values $C_{\max }$ and $C_{\min }$ with a period $T_{e}=$ $1 / f_{e}$ (where $f_{e}$ is the frequency), and we suppose that the function $C_{t}(t)$ has only one local maximum and minimum over a period.

\section{A. Idealized Circuit}

An idealized conditioning circuit implementing a rectangular QV cycle is given in Fig. 3(a). The voltage sources are related as $V_{\text {res }} \leq V_{s t}$ and the diodes are supposed ideal with zero threshold. Let us analyze the evolution of the electrical state of the transducer starting from the moment at which $C_{t}=C_{\text {max }}$, and the transducer voltage $V_{t}$ is $V_{r e s}$, D1 being on. The charge on $C_{t}$ is $Q_{1}=C_{\text {max }} V_{r e s}$. As the transducer capacitance reduces because of the motion of the mobile mass, its voltage increases, switching off the diode $\mathrm{D} 1$, and the voltage across $C_{t}$ increases as:

$$
V_{t}(t)=\frac{Q_{1}}{C_{t}(t)}
$$

The transducer capacitance decreases until $C_{t}=C_{m i n}$; if there exists a capacitance value $C^{*}$ such that $C_{\min } \leq C^{*} \leq C_{\max }$ and such that:

$$
\frac{Q_{1}}{C^{*}}=V_{s t},
$$

the diode D2 turns on before $C_{t}$ reaches $C_{\text {min }}$, and as $C_{\text {min }} \leq C_{t} \leq C^{*}$, the diode D2 is on, and $V_{t}=V_{s t}$. During this phase, the transducer gives charges and energy to the source $V_{s t}$. When $C_{t}$ reaches $C_{m i n}$, the charge on the transducer is $Q_{2}=C_{m i n} V_{s t}$.
When $C_{t}$ starts to increase, the reverse process takes place. First, both diodes are off, and the voltage across $C_{t}$ decreases as

$$
V_{t}(t)=\frac{Q_{2}}{C_{t}(t)}
$$

If there exists a capacitance value $C^{* *}$ such that $C_{\min } \leq C^{* *} \leq C_{\max }$, and such that

$$
\frac{Q_{2}}{C^{* *}}=V_{r e s}
$$

the diode D1 turns on before $C_{t}$ reaches $C_{\max }$, and as $C^{* *} \leq C_{t} \leq C_{\text {max }}$, the diode D2 is on, and $V_{t}=V_{\text {res }}$. During this phase, the transducer collects charges from the source $V_{\text {res }}$.

The described process, when $C_{t}$ goes from $C_{t}=C_{\text {max }}$ to $C_{t}=C_{\min }$ and back to $C_{t}=C_{\max }$, defines a conversion cycle. The corresponding QV diagram is given in Fig. 3(b). In summary, during one cycle the transducer operates twice with a fixed charge, $Q_{1}$ and $Q_{2}$, and twice biased by a constant voltage, $V_{\text {res }}$ and $V_{\text {st }}$.

The energy converted by the transducer during such a cycle is immediately calculated from the QV diagram, as the area of the cycle (Fig. 3(b)):

$$
\begin{aligned}
\Delta W & =\left(V_{s t}-V_{\text {res }}\right)\left(Q_{1}-Q_{2}\right) \\
& =V_{\text {res }}^{2} C_{\text {max }}\left(\frac{V_{s t}}{V_{\text {res }}}-1\right)\left(1-\frac{\frac{V_{s t}}{V_{\text {ress }}}}{\frac{C_{\text {max }}}{C_{\text {min }}}}\right) .
\end{aligned}
$$

The energy $\Delta W$ is not negative if and only if $Q_{1} \geq Q_{2}$, or

$$
\frac{C_{\text {max }}}{C_{\text {min }}} \geq \frac{V_{s t}}{V_{\text {res }}} \text {. }
$$

If this condition is not fulfilled, the capacitance variation is not large enough to allow the amplitude of the $V_{t}$ variation to overcome the potential gap $V_{s t}-V_{\text {res }}$. In this case the transducer operates having a constant charge which depends on its initial condition. The corresponding QV cycle is a horizontal line, having a zero area and hence zero converted power.

The values $C_{\min }$ and $C_{\max }$ are strongly linked to the parameters of the device and to the mechanical mode, and may often be considered as imposed by a given design or application context. The converted energy depends on the extreme values of the transducer capacitance and on the voltages $V_{\text {res }}$ and $V_{s t}$. The latter depend on the practical implementation of the sources. This is discussed in Section III-D.

Since the goal of KEHs is a maximization of the converted energy, one may wonder what the optimal values of the pair $\left(V_{r e s}, V_{s t}\right)$ achieving it are. From (5), one can see that the pair $\left(V_{r e s}, V_{s t}\right)$ does not have an optimal value maximizing the converted energy $\Delta W$. Nevertheless, if one of these voltages are fixed (cf. Section II-B1), there exists an optimal value for the second voltage. If $V_{s t}$ is fixed, the optimal value for $V_{r e s}$ is given by:

$$
\frac{V_{\text {res opt }}}{V_{s t}}=\frac{1}{2}\left(\frac{C_{\min }}{C_{\max }}+1\right) \approx\left[\text { if } \frac{C_{\max }}{C_{\min }} \gg 1\right] \approx \frac{1}{2}
$$

and the corresponding converted energy is

$$
\begin{aligned}
\Delta W_{\max }^{s t} & =\frac{1}{4} V_{s t}^{2} \frac{C_{\min }^{2}}{C_{\max }}\left(\frac{C_{\max }}{C_{\min }}-1\right)^{2} \\
& \approx\left[\text { if } \frac{C_{\max }}{C_{\min }} \gg 1\right] \approx \frac{1}{4} V_{s t}^{2} C_{\max }
\end{aligned}
$$




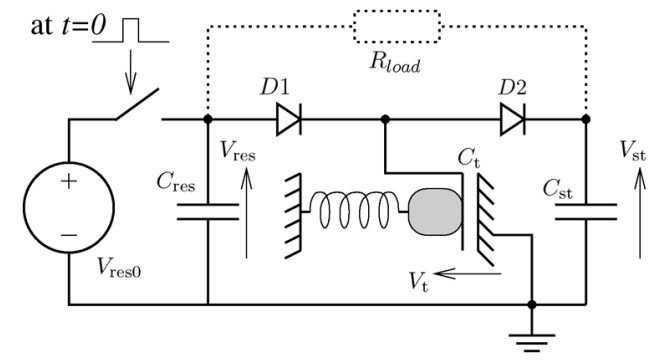

Fig. 4. Schematic of a practical charge pump used for the conditioning of capacitive transducers in KEHs. Dotted lines show the configuration with a resistive flyback, cf. Section III-A.

If $V_{r e s}$ is fixed, the optimal $V_{s t}$ is calculated as:

$$
\frac{V_{\text {st opt }}}{V_{\text {res }}}=\frac{1}{2}\left(\frac{C_{\max }}{C_{\min }}+1\right) \approx\left[\text { if } \frac{C_{\max }}{C_{\min }} \gg 1\right] \approx \frac{1}{2} \frac{C_{\max }}{C_{\min }}
$$

and the optimal energy is

$$
\begin{aligned}
\Delta W_{\max }^{\text {res }} & =\frac{1}{4} V_{\text {res }}^{2} C_{\min }\left(\frac{C_{\max }}{C_{\min }}-1\right)^{2} \\
& \approx\left[\text { if } \frac{C_{\max }}{C_{\min }} \gg 1\right] \approx \frac{1}{4} V_{r e s}^{2} C_{\max } \frac{C_{\max }}{C_{\min }} .
\end{aligned}
$$

It is now interesting to compare the energy converted in one rectangular cycle with the energy converted in a constant voltage triangular $\mathrm{QV}$ cycle $\mathrm{OAB}$, Fig. 2. If the voltage of the available source is $V_{\max }$, the energy is given by [6]:

$$
\begin{aligned}
\Delta W_{\text {const } V} & =\frac{1}{2} V_{\max }^{2} C_{\min }\left(\frac{C_{\max }}{C_{\min }}-1\right) \\
& \approx\left[\text { if } \frac{C_{\max }}{C_{\min }} \gg 1\right] \approx \frac{1}{2} V_{\max }^{2} C_{\max } .
\end{aligned}
$$

A meaningful comparison of these two circuits is possible, if the technology limits the maximal voltage supported by the diodes and the transducer to $V_{\max }$. In this case, the maximal energy obtained by the charge pump is given by (8), in which $V_{s t}$ is substituted with $V_{\max }$. As it can be seen from Fig. 2, the optimal rectangular QV cycle produces less energy than the constant voltage cycle. In the best case, when $C_{\max } \gg C_{\min }$, the ratio is $1 / 2$.

\section{B. Practical Implementation}

1) Circuit Structure and Operation: The network implementing the schematic of Fig. 3(a) was proposed by Roundy et al. [8]. It is shown in Fig. 4, with solid lines. The voltage source $V_{\text {res }}$ is implemented with a large reservoir capacitor, whose value is large enough to neglect its voltage variation over one capacitance variation period. The voltage source $V_{s t}$ is implemented by a storage capacitor $C_{s t}$, which is small compared to $C_{r e s}$, but much greater than the maximum value of the transducer capacitance $C_{\text {max }}$. $C_{\text {res }}$ capacitor is initially charged to a voltage $V_{\text {res }}=V_{\text {res } 0}$, and so are $C_{s t}$ and $C_{t}$ capacitors, because of the charge sharing through the diodes D1 and D2.

In this way, by initially precharging the circuit to a voltage $V_{\text {res } 0}$, we implement the configuration of Fig. 3(a) with $V_{\text {res }}=$ $V_{s t}$, in which the converted energy per cycle should be zero. However, since the $V_{s t}$ voltage source is implemented by a capacitor $C_{s t}$, the charge transfer from $C_{r e s}$ to $C_{s t}$ during the first cycle slightly increases the voltage across $C_{s t}$. Hence, the corresponding QV cycle $D B E$ is actually triangular, as shown in Fig. 5 . The next cycle starts in a configuration with a slightly

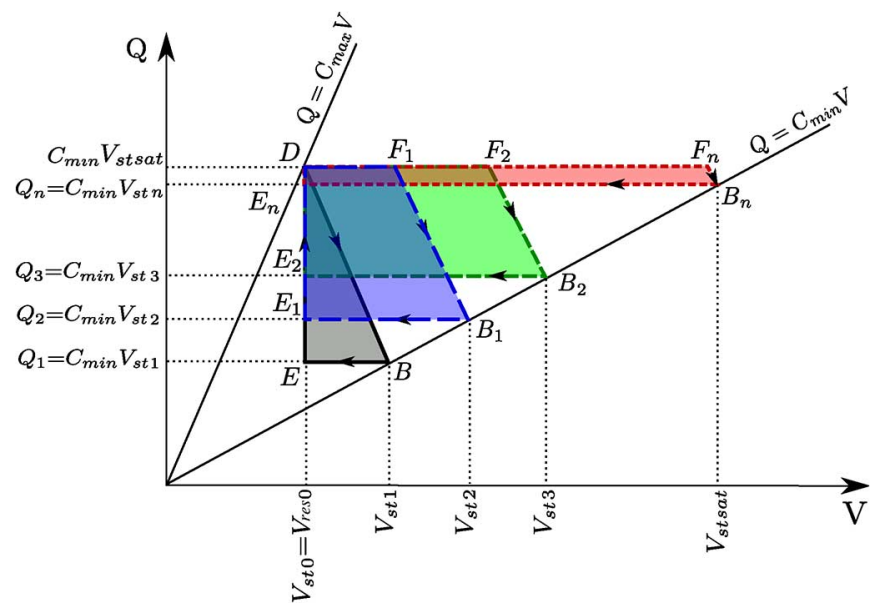

Fig. 5. Succession of charge-voltage cycles as the charge pump operates starting from the state when $V_{s t}=V_{\text {res }}$ : the first cycle $D B E$, the subsequent cycles $D F_{1} B_{1} E_{1}, D F_{2} B_{2} E_{2}, \ldots, D F_{n} B_{n} E_{n}$. If $n \rightarrow \infty$, the cycle is degenerated to a line passing through the points $D, F_{1}, F_{2} \ldots F_{n}$.

larger $V_{s t}$, yielding a trapezoidal QV cycle. After each cycle, $V_{s t}$ increases, so that all further cycles are different. It can be shown that the slope of the line $D B, F_{1} B_{1}, \ldots F_{n} B_{n}$ is $-C_{s t}$ (steeper than $C_{\text {max }}$, but exaggerated on the figure): the shape of the cycles is close to rectangular, as $C_{s t} \gg C_{\max }$. It can be seen that the QV trajectory follows a succession of cycles, starting from $V_{s t} \approx V_{\text {res }}$ and asymptotically approaching the degenerated cycle corresponding to $V_{\text {st sat }}=V_{\text {res }} C_{\text {max }} / C_{\text {min }}$. This value is called the "saturation voltage" of the charge pump.

As a result of the operation of this circuit, the capacitor $C_{s t}$ receives energy from $C_{r e s}$, and in addition, it receives energy converted from the mechanical domain (the harvested energy). In this way, at every QV cycle of the charge pump operation, the overall electrical energy of the circuit increases. In what follows, we present a quantitative analysis of this process.

The voltage $V_{s t}$ at the end of the $n$th cycle evolves following the recursive equation [10]:

$$
V_{\text {st } n}=\alpha V_{\text {st } n-1}+\beta,
$$

where

$$
\alpha=\frac{C_{s t}}{C_{s t}+C_{\min }}, \quad \text { and } \quad \beta=V_{\text {res }} \frac{C_{\max }}{C_{s t}+C_{\min }} .
$$

The solution of the discrete equation (12) is:

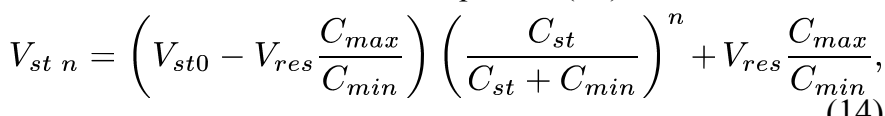

where $V_{s t 0}$ is the voltage on $C_{s t}$ capacitor at the zeroth cycle. In this article, it is assumed $V_{s t 0}=V_{\text {res } 0}$.

Note that if $C_{t}$ variations are periodic, the variable $n$ is the operating time of the charge pump divided by the period of $C_{t}$ variation, $T_{e}$. It is easy to see that the asymptotic value of $V_{s t} n$ as $n \rightarrow \infty$ is $V_{\text {st sat }}$.

As $V_{s t}$ increases, the voltage $V_{\text {res }}$ slowly decreases, as the amount of charges on $C_{r e s}$ and $C_{s t}$ is constant (as one neglects the charges at $C_{t}$ ):

$$
V_{\text {res } n}=V_{\text {res } 0}-\left.\frac{C_{\text {st }}}{C_{\text {res }}} \cdot\left(V_{\text {st } n}-V_{\text {res } 0}\right) \approx V_{\text {res } 0}\right|_{\text {if } C_{\text {res }} \gg C_{s t}} .
$$

An example of the evolution of $V_{\text {res }}$ and $V_{s t}$ is given in Fig. 6(a), where the charge pump starts from the state $V_{\text {res }}=V_{s t}=V_{t}=V_{\text {res } 0}$. From formula (12) it can be seen 

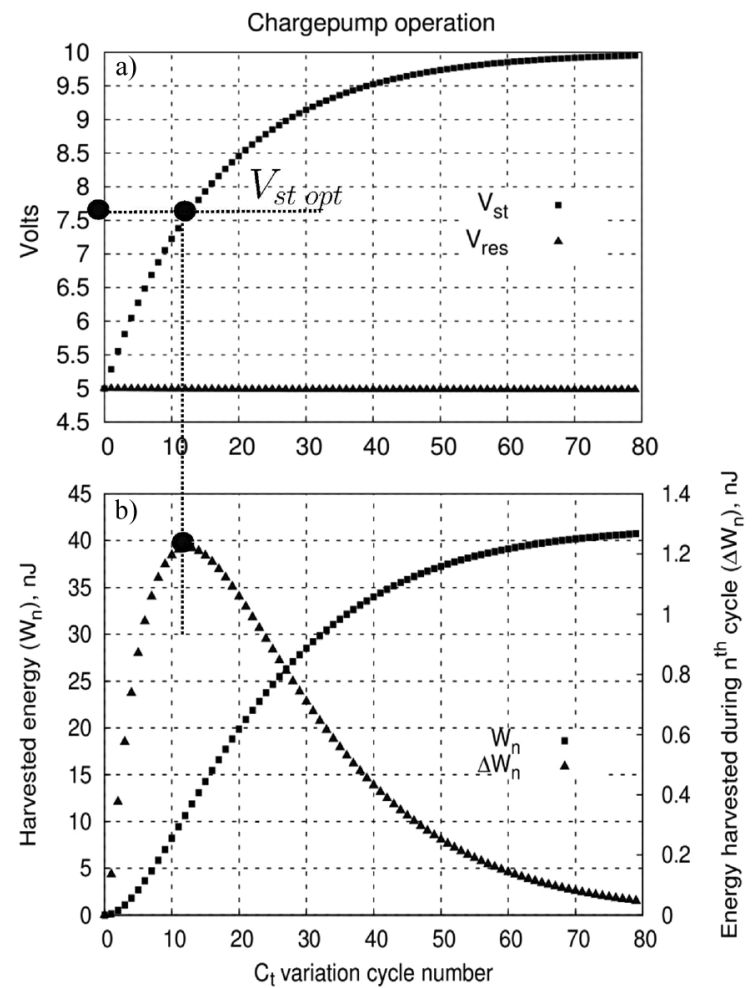

Fig. 6. Evolution of the electrical state of the charge pump without flyback. a) Evolution of $V_{s t}$ and $V_{\text {res }}$ voltages, b) Evolution of the total converted energy $\left(W_{n}\right)$ and of the energy converted at one cycle $\left(\Delta W_{n}\right)$. The simulation was done with $C_{\min }=200 \mathrm{pF}, C_{m a x}=400 \mathrm{pF}, C_{s t}=3.3 \mathrm{nF}, C_{r e s}=\infty$, $f_{e}=300 \mathrm{~Hz}$.

that if $C_{s t} \gg C_{\max }$, the increment of $V_{s t}$ during every cycle is small, and the charge pump run through cycles for all possible $V_{s t}$, from $V_{\text {res } 0}$ to the saturation value $V_{\text {st } s a t}$.

2) Evolution of the Harvested Energy: Neglecting the energy in the transducer capacitance since it is small, and supposing that the $C_{s t}$ voltage is initially $V_{\text {res } 0}$, the energy harvested during $n$ first vibration periods is stored in the capacitors $C_{r e s}$ and $C_{s t}$, and is given by:

$$
W_{n}=\frac{1}{2} C_{s t} V_{s t n}^{2}+\frac{1}{2} C_{r e s} V_{r e s n}^{2}-\frac{1}{2}\left(C_{r e s}+C_{s t}\right) V_{r e s 0}^{2},
$$

where $V_{\text {res } n}$ is the $C_{\text {res }}$ voltage after $n$ vibration cycles, $V_{\text {res } 0}$ is the initial voltage of $C_{r e s}$. At the same time, the sum of charges of $C_{s t}$ and $C_{r e s}$ is constant $\left(Q_{0}\right)$, so, the following relation holds (neglecting the charge sharing with $C_{t}$ ):

$$
Q_{0}=\left(C_{s t}+C_{r e s}\right) V_{r e s 0}=C_{s t} V_{s t n}+C_{r e s} V_{\text {res } n} .
$$

Using (17), (16) can be simplified:

$$
W_{n}=\frac{\frac{C_{s t} C_{r e s}}{C_{s t}+C_{r e s}}\left(V_{s t n}-V_{\text {res } n}\right)^{2}}{2} .
$$

This shows that the harvested energy is stored in the capacitor composed of $C_{s t}$ and $C_{r e s}$ connected in series. This formula can be further simplified if $C_{s t} \ll C_{r e s}$ and if $C_{r e s}$ is large, so that $V_{\text {res }} \approx V_{\text {res } 0}$. In this case:

$$
W_{n} \approx \frac{C_{s t}\left(V_{s t n}-V_{r e s 0}\right)^{2}}{2} .
$$

This equation shows that the converted energy is mainly stored in the $C_{s t}$ capacitor.

The value

$$
\Delta W_{n}=W_{n}-W_{n-1}
$$

gives the energy converted during one cycle. For the case when $C_{s t} \gg C_{\text {max }}$, this quantity can also be found from (5) by substituting $V_{s t}=V_{s t n-1}$, and $V_{\text {res }}=V_{\text {res } 0}$.

Equation (19) relates the harvested energy only with the voltages $V_{\text {res }}$ and $V_{s t}$, hiding the operation by which the charge pump came to this state. It is thus possible to consider $V_{r e s}$ and $V_{s t}$ as "macroscopic state parameters" of the charge pump, remove the indices $n$ and write:

$$
W\left(V_{r e s 0}, V_{s t}\right)=\frac{C_{s t}\left(V_{s t}-V_{r e s 0}\right)^{2}}{2} .
$$

If, during the operation of the charge pump, the $V_{s t}$ voltage increases from some $V_{1}$ to some $V_{2}$, the corresponding converted energy is given by:

$$
\begin{aligned}
\Delta W\left(V_{\text {res } 0}, V_{1}, V_{2}\right) & =W\left(V_{\text {res } 0}, V_{2}\right)-W\left(V_{\text {res } 0}, V_{1}\right) \\
& =\frac{C_{s t}\left(V_{2}-V_{1}\right)\left(V_{2}+V_{1}-V_{r e s 0}\right)}{2} .
\end{aligned}
$$

Fig. 6(b) presents the plots characterizing the energy conversion by the charge pump. During every cycle $n$, the total energy (lower plot, square dots) increases by the value given by (5) calculated for the $V_{s t}$ resulting from the previous cycle (upper plot, squares dots). The energy $\Delta W_{n}$ converted at each cycle is presented by the plot with triangular dots. Only a few cycles convert a significant amount of energy: those corresponding to $V_{s t}$ values close to the optimal $V_{s t ~ o p t}$, (cf. the analysis of Section II for fixed $\left.V_{r e s}\right)$. This value is situated in-between the extreme values of $V_{s t}$, and a freely running charge pump finishes by entering into the saturation mode, in which $V_{s t} \approx V_{\text {st sat }}$ and $\Delta W \approx 0$.

\section{Shortcomings of the Primitive Circuit}

Because of the saturation, the charge pump alone is not useful for capacitive KEHs. Practical conditioning circuits should be able to i) ensure a sustainable energy conversion, ii) be able to accumulate the converted energy. These two very important points are discussed in this subsection.

1) Need for a Flyback: The plot $\Delta W_{n}$ in Fig. 6(b) can be seen as the average power converted in a cycle. The goal of a harvester is to permanently maintain the converted power close to a maximum level. For this reason, a charge pump needs periodic flyback: a mechanism returning some of charges from $C_{s t}$ to $C_{r e s}$ so as to keep $V_{s t}$ close to the optimal value and to prevent the charge pump from saturation. Possible implementations of the flyback will be discussed in the next two sections.

The action of the charge pump can be seen as the generation of an average current flowing from a low potential $\left(V_{\text {res }}\right)$ to a higher potential $V_{s t}$. This current is roughly equal to

$$
i_{\text {ch } \text { pump }}=\frac{Q_{1}-Q_{2}}{T_{e}}=\frac{V_{r e s} C_{m a x}-V_{s t} C_{m i n}}{T_{e}} .
$$

The flyback must create an opposite current from $C_{s t}$ to $C_{r e s}$, preventing the accumulation of charges on $C_{s t}$, and so, avoiding the charge pump saturation. This current, flowing from the high to low potential, removes the electrical energy from the charge pump, and in a practical application, this energy should be transferred to a useful load. In the steady-state mode, the rate at which the energy is removed from the charge pump is equal to the power of the electromechanical conversion.

2) Auto-Increasing of the Internal Energy: From (5) and further analysis of Section II-B1, it can be seen that the energy converted by a charge pump is proportional to the square of the 
voltage $V_{s t}$ and $V_{\text {res }}$, i.e., proportional to the internal energy of the circuit in Fig. 4. Practical studies highlight that up to tens of volts may be needed for optimal operation [11]. However, the initial charge can only be obtained by a low-voltage battery existing in the system, or by a complementary piezoelectric $\mathrm{KEH}$ as proposed in [12]. In both cases, the initial voltage generated in this way will certainly be low (few volts).

By consequence, the conditioning circuit should be able to use a part of the converted energy in order to increase its biasing: its internal energy represented by the voltage on the largest capacitor, $V_{r e s}$. We call this property "auto-increasing of the internal energy," and the corresponding operation mode "accumulative mode." This mode allows not only a definition of high initial bias, but also a compensation of eventual losses in the conditioning circuit (leakages in the capacitors).

The auto-increasing capability is not offered by the basic charge pump.

\section{Circuits Derived From the Primitive Charge PumP}

\section{A. Resistive Flyback}

The simplest way to implement a flyback is to connect a load between $C_{r e s}$ and $C_{s t}$ (Fig. 4), originally proposed in [12]. Here, the load is represented with a resistance $R_{\text {load }}$ whose current is:

$$
i_{\text {load }}=\frac{V_{\text {st }}-V_{\text {res }}}{R_{\text {load }}} .
$$

A simplified analysis may be done by analyzing independently the charge flows of the charge pump and of the resistor, and then by superimposing them. The point at which both currents are equal to each other $\left(i_{\text {ch pump }}=i_{\text {load }}\right)$ corresponds to steadystate operation. Although it is an approximation based on the averaging method [13], it gives good analytical results.

In the steady state, the average $V_{s t}$ voltage on a period $T_{e}$ is the same for all cycles. The stability of this mode can be easily proven by supposing a perturbation which yields, for example, $i_{\text {ch pump }}<i_{\text {load }}$. In this case, the load current consumption outweighs the current due to the charge generation by the pump, and $V_{s t}$ decreases. This results in decreasing of $i_{\text {load }}$, and so in reducing the initial perturbation.

Under these considerations, the average steady-state voltage $V_{s t}$ with resistive flyback can be predetermined by equating (23) and (24). Conversely, the value of $R_{\text {load }}$ yielding a desired $V_{s t}$ (for example, that yielding a maximal $\Delta W$ ) can be calculated.

The resistive flyback configuration provides a sustainable energy conversion by preventing the charge pump from the saturation. This is an efficient solution from the point of view of the simplicity of implementation. However, it does not allow an auto-increasing of its internal energy (cf. Section II-C2), and for this reason, is not usable in practical applications.

\section{B. Inductive Flyback}

1) Architecture and Operation: The inductive flyback is a Buck DC-DC converter, transferring energy from $C_{s t}$ to $C_{r e s}$ (Fig. 7(a)) [10]. The flyback operates in two steps:

i) When $V_{s t}$ is high so that the efficiency of the charge pump decreases, some external control device (cf. the explanation below) activates the switch $S W$, and the current in the inductor $L$ starts to increase. This results in: i) charges
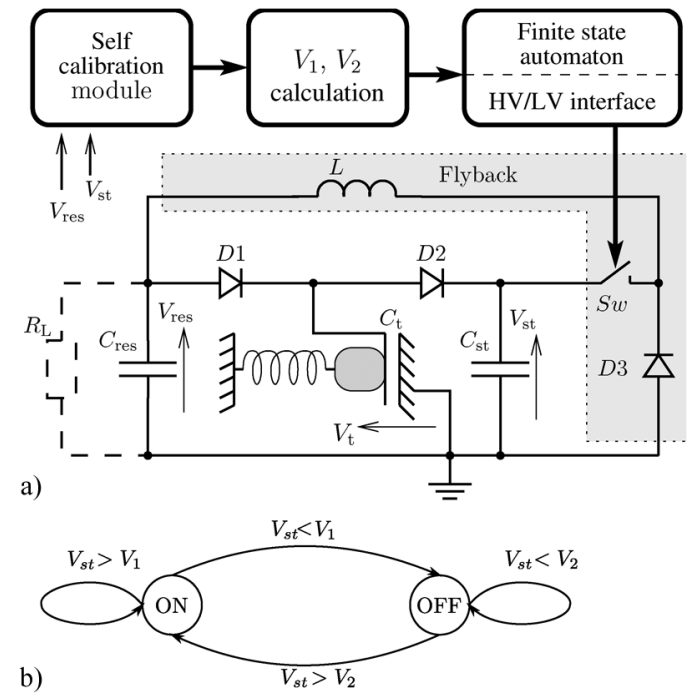

Fig. 7. Conditioning circuit with inductive flyback. (a) The architecture of the circuit: the basic charge pump (as presented in Section II-B), the inductive flyback (grey background, Section III-B), and the architecture of the adaptive control (cf. Section III-B), (b) the finite state automaton explaining the algorithm of the flyback switch control.

being transferred from $C_{s t}$ to $C_{r e s}$, so that $V_{s t}$ decreases, ii) the energy previously generated by the charge pump on $C_{s t}$ is transferred to $L$.

ii) As the magnitude of $V_{s t}$ decreases, it becomes close to $V_{\text {res }}$, and the same external device cuts the switch, so that the inductor discharges through the flyback diode $D 3$ onto $C_{r e s}$. This results in a transfer of the inductor energy to $C_{r e s}$, and an increase of the $C_{r e s}$ voltage, since the diode $D 3$ is connected to the ground. In this way, the converted energy is used to extract charges from the ground and inject new charges into the charge pump.

It can be seen that $C_{r e s}$ receives charges twice: in the first phase, it receives the charges previously given to $C_{s t}$ during the pump operation, and in a second phase, it receives new charges from the ground. In this way, $C_{r e s}$ receives the energy it has given to $C_{s t}$ during the operation of the charge pump, and it receives the energy converted from the mechanical domain. If such an operation is cyclic, the average voltage and energy of $C_{r e s}$ will grow. In this way, two problems are solved: i) even if the capacitors are leaky, the losses are compensated by the charges extracted from the ground, ii) a large capacitor $C_{r e s}$ contains the harvested energy available for the load (represented by dotted lines in Fig. 7(a)). If no load is present, $C_{\text {res }}$ accumulates the converted energy, and so implements an "auto-increasing" or "accumulative" mode.

The advantage of the charge pump with inductive flyback is the possibility of a precise control of the QV cycle corresponding to the energy conversion. This is achieved by a modulation of the average $V_{s t}$ voltage so as to remain in the optimal mode for energy conversion.

A drawback of the circuit is the need of an external command for the switch. However, this command is synchronized not with the mobile mass vibrations, but with the evolution of the voltage $V_{s t}$ : firstly, a voltage can easily be measured, and secondly, the evolution is low compared to the period of the capacitance variation. Hence, the cost of such a command is smaller than for circuits implementing constant-voltage or constant-charge QV cycles (cf. Section I). 


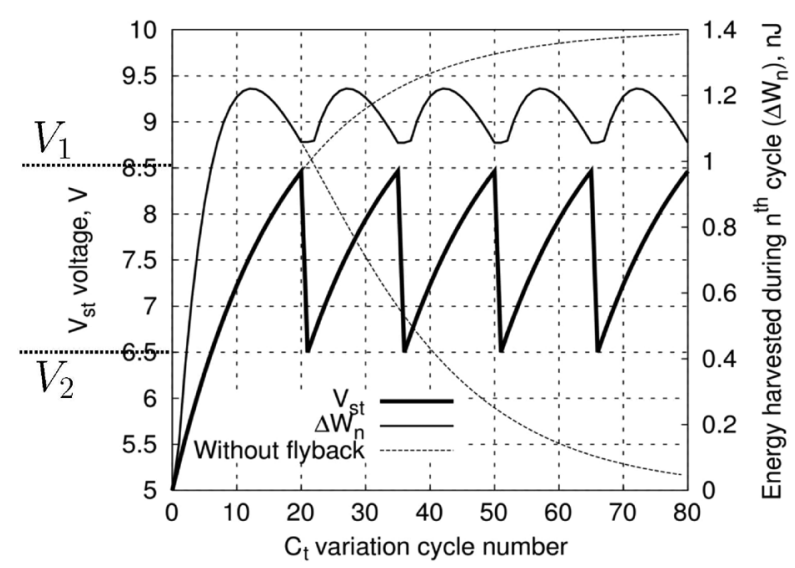

Fig. 8. Evolution of dynamic quantities of the charge pump with inductive flyback. The parameters of the circuit used in the simulation are the same as for Fig. 6 .

2) Practical Implementation: In order to take maximum advantage of the charge pump with inductive flyback, a "smart" management of the flyback is required (cf. functional representation in Fig. 7(a)). This was firstly highlighted in [14]. The most important issue is the selection of an interval $\left[V_{1}, V_{2}\right]$ for $V_{s t}$ values, so that $V_{1}<V_{\text {st opt }}<V_{2}$, and $V_{1}$ and $V_{2}$ are close to $V_{\text {opt }}$. As far as $V_{1}$ and $V_{2}$ are chosen, the operation of the switch is ordered by a finite-state machine (Fig. 7(b)), and the evolution of $V_{s t}$ and the average power is given in Fig. 8.

The voltage $V_{1}$ and $V_{2}$ may be defined as $V_{\text {st opt }} \pm \Delta V$, where $\Delta V$ is chosen large enough so to minimize the frequency of the switch activation, but at the same time small enough to keep the average power close to the maximal value. By considering (14), the reader can verify that the value of the capacitance $C_{s t}$ controls the number of cycles during which the voltage $V_{\text {st }}$ goes from $V_{1}$ to $V_{2}$, and at the same time, the energy that the flyback circuit must transfer from $C_{s t}$ to $C_{r e s}$. A trade-off must be found, as a function of the practical implementation of the whole system.

However, $V_{\text {st opt }}$ is not directly measurable, and this is a fundamental problem. From (9), it can be seen that $V_{\text {st opt }}$ depends on $C_{\max } / C_{\min }$, which is not known a priori in practice, as it depends on the amplitude of the mobile mass vibration (cf. Part 2 of this paper). One of the possible ways to determine $V_{s t ~ o p t}$ is to measure the saturation voltage of a freely run charge pump. This voltage is given by $V_{s t ~ s a t}=V_{\text {res }} C_{\text {max }} / C_{\text {min }}$. As it can be seen in Fig. 8, the values of $V_{1}$ and $V_{2}$ can just be chosen in-between $V_{\text {res }}$ and $V_{\text {st sat }}$, for example,

$$
\begin{aligned}
& V_{1}=V_{\text {res }}+1 / 3 \cdot\left(V_{\text {st sat }}-V_{\text {res }}\right), \\
& V_{2}=V_{\text {res }}+2 / 3 \cdot\left(V_{\text {st sat }}-V_{\text {res }}\right)
\end{aligned}
$$

As far as $V_{\text {st sat }}$ is known, the calculation on $V_{1}, V_{2}$ may be done by simple analog circuits, such as a voltage divider [15]. To determine $V_{\text {st sat }}$, the charge pump should be run up to saturation periodically, and the final $V_{s t}$ value should be measured and memorized. This measurement should be periodic, since the vibration amplitude may change with time. We call this technique "auto-calibration," since a periodic re-calculation of $V_{1}$ and $V_{2}$ allows an optimal operation of the circuit for a range of parameters of external vibrations.

Practical implementation of adaptive architecture in Fig. 7 is challenged by the relatively low power available for the control

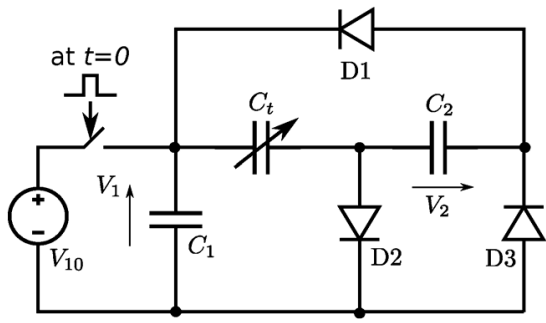

Fig. 9. Original schematic of conditioning circuit based on the Bennet's doubler [18].

circuitry, and by the need to manage high voltages in the conditioning circuit. Most studies used an "old" CMOS technology supporting high voltages (e.g., $0.7 \mu \mathrm{m}$ CMOS in [4]). The work [16] used a mixed high-voltage/low-voltage $0.35 \mu \mathrm{m}$ CMOS technology, where high voltage circuits are used for the interface with the charge pump, and the processing is done by low voltage parts. This allows a minimization of the power overhead of the control electronics. Implementation of a high-side high voltage MOS switch for the flyback control with a low voltage control interface represented a particular difficulty. An advanced study of a fully integrated CMOS implementation of the control architecture, as in Fig. 7, was presented in [15], [16]. Although the full IC was not implemented, the circuit was designed up to the layout. Transistor-level simulations highlight a power consumption of the overall smart adaptive circuit with self-calibration of less than $1.5 \mu \mathrm{W}$.

\section{Bennet's Doubler}

First introduced at the end of the 18th century [17], the Bennet's doubler of electricity has recently been proposed by Queiroz [18] for capacitive KEH (cf. Fig. 9). Since then, several works have further developed this concept, adapting it to microscale KEH [19]-[21].

1) Basic Configuration: Detailed analysis of the mechanism of the energy conversion in the circuit of Fig. 9 has been presented in the papers cited above. Here, emphasis is made on the similarity between this circuit and an idealized charge pump (Fig. 3(a)), and on the application of the analysis developed in Section II-A.

Let's consider $V_{10}$ as a positive voltage source that can be used to pre-charge $C_{1}$ when desired. When applied, it also charges $C_{t}$ to $V_{10}$ through D2. Then, for several cycles, thanks to the capacitance variation that allows the circuit to overcome the diodes potential gaps, $C_{1}$ charges $C_{2}$ through $C_{t}$ which acts as a charge buffer: when $C_{t}$ goes to $C_{\text {max }}$, it absorbs charges from $C_{1}$, and when it goes to $C_{m i n}$, the energy of the charges is elevated and the charges are released to $C_{2}$. At the end of this transient process, the voltages on capacitors $C_{1}$ and $C_{2}$ are equal, with polarities as indicated in Fig. 9. A more detailed description of the transient process for the Bennet's doubler can be found in [21].

After the transient, the circuit is in the energy conversion mode, which will be referred to as the steady-state operation mode of the circuit. As shown in [18], this mode shows an avalanche-like growth of the circuit voltages: the energy converted by the circuit in one cycle is distributed among the circuit capacitors, and so increases the circuit initial bias for the next cycle. Since the energy converted in a cycle is proportional to the square of the voltages, both the voltages and the energy in 


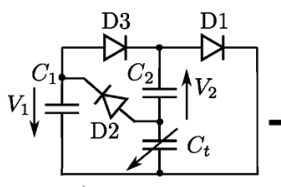

a)

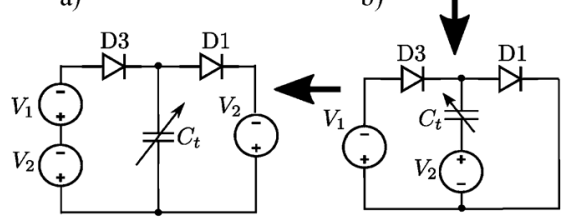

c)

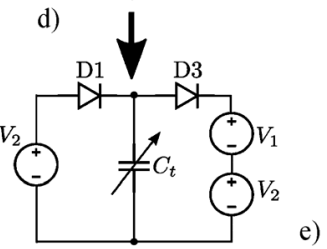

e)

Fig. 10. Demonstration of similarity of the Bennet's doubler operation in the steady-state mode with basic charge pump (Fig. 3(a)): (a) circuit in Fig. 9 redrawn, (b) the same topology presented in steady-state mode without D2 and where the fixed capacitors are replaced by voltage sources; (c) the same circuit with $V_{2}$ source and $C_{t}$ swapped, since they are in series, (d) the source $V_{2}$ "passes" through the bottom node and appears in the left and right side branches, (e) the same as (d) redrawn. The topology (e) is equivalent to that of Fig. 3(a) if $V_{\text {res }}=V_{2}, V_{s t}=V_{1}+V_{2}$.

the circuit's capacitors grow exponentially. The steady-state operation is an auto-increasing mode (discussed in Section II-C2), which, contrarily to the charge pump with inductive flyback, does not require an external control.

A similarity between the Bennet's doubler in the steady-state mode and the basic configuration of the charge pump (Fig. 3(a)) can be exposed if the circuit is redrawn as in Fig. 10(a). It can be shown that the conduction of D2 in the steady-state mode is negligible, if $C_{1} \gg C_{2}$ (cf. [18]). If $\mathrm{D} 2$ is removed and if the fixed capacitors are represented by voltage sources (valid if they are much greater than $C_{\max }$ ), then by equivalent circuit transformations presented in Fig. 10(b)-(d) one obtains an equivalent topology given in Fig. 10(e). The similarity with the network of Fig. 3(a) is obvious if one puts $V_{\text {res }}=V_{2}, V_{s t}=V_{1}+V_{2}$. Details of the equivalent circuit transformation are given in the legend of Fig. 10.

The QV cycle corresponding to the circuit (Fig. 11) has the parameters:

$$
\begin{aligned}
V_{\text {res }} & =V_{2} \\
V_{s t} & =V_{1}+V_{2}
\end{aligned}
$$

and since $V_{1}=V_{2}$, for this configuration, the relation $V_{\text {store }} / V_{\text {res }}$ is fixed as a ratio of 2 by the topology (Fig. 11(a)). The main shortcoming of the basic configuration of the Bennet's doubler is that the ratio $C_{\max } / C_{\min }$ must be at least 2 for the operation of the circuit (cf. condition (6)), and transducers implemented in MEMS technology often display lower performances.

2) Generalization of the Circuit: Recent studies highlighted that ratios $V_{\text {st }} / V_{\text {res }}$ lower than 2 can be achieved by a modification of the circuit topology. In [19], a voltage multiplier is used in order to define a ratio between $V_{1}$ and $V_{2}$ as:

$$
V_{2}=k V_{1}
$$

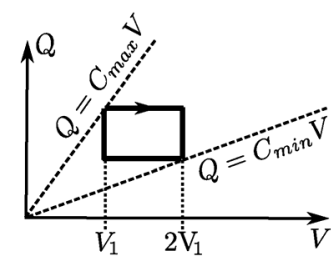

a)

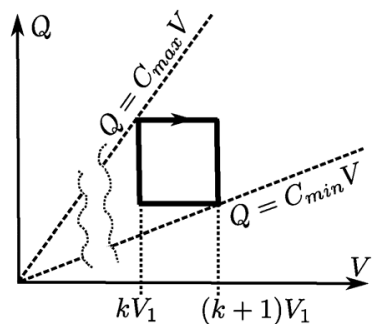

b)
Fig. 11. (a) QV cycle diagram for the basic configuration of Bennet's doubler [18], QV cycle diagram for the more general Bennet's doubler [19].

$k \geq 2, k \in \mathbb{N}$. The previous analysis done on the basic Bennet's circuit is still valid; the QV cycle is given by substituting (27) into (26).

This allows one to consider a generalized Bennet's doubler circuit, in which the ratio between $V_{1}$ and $V_{2}$ can be any value. To date, only topologies with integer ratios have been implemented, but further investigation may result in the discovery of configurations with non-integer rational ratios. In general, for any $V_{1}$ and $V_{2}$ values such that $V_{1} \leq V_{2}$, the energy converted in a cycle by a general Bennet's doubler is obtained by combining (5) and (26):

$$
\Delta W=V_{1} V_{2} C_{\max }\left(1-\frac{V_{1} / V_{2}+1}{\frac{C_{\max }}{C_{\min }}}\right)
$$

The circuit generates electricity as long as $\Delta W$ in (28) is positive, i.e., when

$$
\frac{V_{1}}{V_{2}}<C_{\max } / C_{\min }-1 .
$$

By consequence, the advantage of the Bennet's doubler with voltages $V_{1}$ and $V_{2}$ given by (27), is a compatibility with transducers having ratio $C_{\max } / C_{\min }$ less than 2 if $k>1$. The corresponding QV cycle is given in Fig. 11.

\section{Comparison of Bennet's Doubler and the Charge Pump}

The energy conversion yield of circuits implementing rectangular QV cycles was discussed in Section II, and the results are fully applicable to both the circuits based on the charge pump and on the Bennet's doubler. It was shown that the efficiency of the conversion is controlled by the ratio $V_{s t} / V_{\text {res }}$, and that the optimization depends on which one of the two voltages is fixed. However, $V_{\text {res }}$ and $V_{s t}$ are defined differently in the two circuits, and a direct comparison can not be done, unless an application case is defined. We propose to study two cases, which are discussed below. The synthesis of this comparison is given in Table I, which expresses the energy converted during one QV cycle by all circuits considered in this paper.

i) The same fixed voltage is applied on the pre-biased capacitor ( $V_{\text {res }}$ in the chargepump, $V_{1}$ in the Bennet's doubler). This is a reasonable assumption from the standpoint of practical applications, since the prebiased capacitor is directly connected to an interface with the load, and its voltage is likely to be limited by the transistor technology. Setting $V_{1}=V_{\text {res }}=V_{0}$, from (28), (27) we obtain the energy converted in one cycle by the Bennet's doubler:

$$
\Delta W=k C_{\min } V_{0}^{2}\left(\frac{C_{\max }}{C_{\min }}-\frac{1}{k}-1\right)
$$


TABLE I

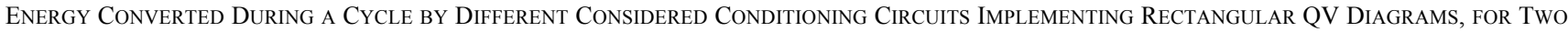
Cases: (I) the Maximal Supportable Voltage is Fixed $\left(V_{m a x}\right)$, (II) the Initial Available Pre-Charge Voltage is Fixed ( $\left.V_{0}\right)$. The Following PARAMETERS ARE USED: $C_{\max } / C_{\min }=\gamma$

\begin{tabular}{|c|c|c|c|c|c|}
\hline \multicolumn{2}{|c|}{ Energy converted by cycle } & Const. Volt. Circuit & Roundy Charge Pump & Bennet Doubler & Bennet Doubler+multiplier \\
\hline \multirow{3}{*}{$\begin{array}{c}V_{0} \\
\text { fixed }\end{array}$} & General formula & $\frac{1}{2} V_{0}^{2} C_{\min }(\gamma-1)^{a}$ & $\frac{1}{4} V_{0}^{2} C_{\min }(\gamma-1)^{2}$ & $C_{\min } V_{0}^{2}(\gamma-2)$ & $k C_{\min } V_{0}^{2}\left(\gamma-\frac{1}{k}-1\right)$ \\
\hline & $\gamma \gg 1$ & $\frac{1}{2} V_{0}^{2} C_{\max }$ & $\frac{1}{4} V_{0}^{2} C_{\max } \gamma$ & $V_{0}^{2} C_{\max }$ & $k C_{\max } V_{0}^{2}$ \\
\hline & $1 \lesssim \gamma<2$ & $\frac{1}{2} V_{0}^{2} C_{\min }(\gamma-1)$ & $\frac{1}{4} V_{0}^{2} C_{\min }(\gamma-1)^{2}$ & Not applicable & $k C_{\min } V_{0}^{2}\left(\gamma-\frac{1}{k}-1\right) b$ \\
\hline \multirow{3}{*}{$\begin{array}{l}V_{\max } \\
\text { fixed }\end{array}$} & General formula & $\frac{1}{2} V_{\max }^{2} C_{\min }(\gamma-1)$ & $\frac{1}{4} V_{\max }^{2} C_{\min } \gamma^{-1}(\gamma-1)^{2}$ & $\frac{1}{4} V_{\max }^{2} C_{\min }(\gamma-2)$ & $\frac{k}{(k+1)^{2}} V_{\max }^{2} C_{\min }\left(\gamma-\frac{1}{k}-1\right)$ \\
\hline & $\gamma \gg 1$ & $\frac{1}{2} V_{\max }^{2} C_{\max }$ & $\frac{1}{4} V_{\max }^{2} C_{\max }$ & $\frac{1}{4} V_{\max }^{2} C_{\max }$ & $\frac{k}{(k+1)^{2}} V_{\max }^{2} C_{\max }$ \\
\hline & $1 \lesssim \gamma<2$ & $\frac{1}{2} V_{\max }^{2} C_{\min }(\gamma-1)$ & $\frac{1}{4} V_{\max }^{2} C_{\min }(\gamma-1)^{2}$ & Not applicable & $\frac{k}{(k+1)^{2}} V_{\max }^{2} C_{\min }\left(\gamma-\frac{1}{k}-1\right)$ \\
\hline
\end{tabular}

${ }^{a}$ For the constant voltage circuit, the available voltage source generates the maximal voltage applied on the transducer during the cycle.

Hence, $V_{0}$ and $V_{\max }$ are the same, and the same formulae are used in the table.

${ }^{b}$ Here it is supposed that $\gamma-1>1 / k$.

The maximal energy produced in a cycle by the charge pump circuit with a fixed $V_{\text {res }}$ is given by (10), where, for the comparison, $V_{r e s}$ should be substituted by $V_{0}$. Let us now compare this maximal energy with the value given for (30), for different $k$.

If $k=1$ (basic Bennet's doubler), the charge pump is advantageous for any value of $C_{\max } / C_{\min }$ (note that for $C_{\text {max }} / C_{\text {min }}<2$, the basic Bennet's doubler does not work, cf. (29)). However, for large $k$ (so that $1 / k$ can be neglected in (30)), the comparison is mitigated: for $C_{\max } / C_{\min }>4 k+1$ the charge pump is more advantageous. However, for the cases where $C_{\max } / C_{\min }$ is small and comparable with unity (which is very common with MEMS devices), the Bennet's doubler with multiplication becomes much more interesting. The price to pay for this improvement is a high maximum voltage $V_{s t}$ which should be supported by the transducer and the diodes: it is equal to $(k+1) V_{0}$.

ii) For this reason, another case is relevant, when the maximum voltage supported by the transducer $\left(V_{s t}\right)$ is fixed to $V_{\max }$ by the transistor/diode technology, or by the limitations of the electromechanical device. The maximal energy provided by the charge pump at fixed $V_{s t}$ is given by (8): For the comparison, $V_{s t}$ should be substituted with $V_{\max }$.

In order to compare the maximal energy yield of the charge pump with that of the Bennet's doubler, $V_{1}$ and $V_{2}$ should be expressed through $V_{\max }$. Setting $V_{s t}=V_{\max }$ in (26), we recalculate the voltages of the Bennet's doubler $V_{1}=V_{\max } /(k+1)$, $V_{2}=k V_{\max } /(k+1)$, and we obtain the energy converted in one cycle:

$$
\Delta W=C_{\min } V_{\max }^{2} \frac{k}{(k+1)^{2}}\left(\frac{C_{\max }}{C_{\min }}-\frac{1}{k}-1\right) .
$$

The comparison shows that a conventional Bennet's doubler $(k=1)$ is less advantageous than the charge pump for $C_{\max } / C_{\min }$ comparable to unity, and for large $C_{\max } / C_{\min }$ they offers equivalent yields. If $k$ is large (so that $k \gg 1$ ), the charge pump is still more efficient for $C_{\max } / C_{\min }>k /(k-4)$, and the Bennet's doubler is only advantageous for $1+1 / k<C_{\max } / C_{\min }<k /(k-4)$.

As a conclusion, the Bennet's doubler has a better power yield for cases when $C_{\max } / C_{\min }$ is small, a voltage multiplier is used and there is no major limitation on the maximum voltage supported by the devices. In all other cases, the charge pump allows a greater or similar converted power. However, the price to pay for this advantage is the need of an external control for the charge pump and of an inductor which is complicated to integrate. For a steady optimal operation, the voltage $V_{s t}$ should be maintained at its optimal value defined by the $C_{\max } / C_{\min }$ ratio, and this costs power and complexity.

\section{EXPERIMENTAL RESULTS}

Several studies have addressed experimental validation of different circuits with rectangular QV cycle. Most of them implemented the self-increasing operation. The study of this "accumulative" (loadless) mode is particularly interesting, since it provides a characterization of the power conversion for a range of the bias voltage. The value of the converted power is given by the rate of the energy evolution on the reservoir capacitor. In the accumulative mode, the circuits highlights an exponential increase of the voltage on $C_{r e s}$ in the charge pump [4], [10], or on $C_{1}$ in the Bennet's doubler [18]. In the experiments where macroscopic variable capacitors are used, this increase of voltage reaches hundreds or thousands of volts, provoking an electrical break-down of the used insulator. Few experiments successfully conditioned microscale MEMS devices with circuits implementing rectangular QV cycles [22], [23]. The diagram of the experiment is presented in Fig. 12. The used MEMS device is a micro resonator associated to a capacitive transducer presented and characterized in [11]. The MEMS device was submitted to sinusoidal external vibrations, with fixed frequency and amplitude.

Both Bennet's doubler (Fig. 9) and charge pump based circuit with inductive flyback (Fig. 7(a)) were implemented with discrete elements, and tested with the above mentioned MEMS transducer. In the charge pump circuit, the flyback switch SW was driven by a fixed periodic sequence. Both circuits, when operating in accumulative mode, demonstrated a similar behavior different from the case when a macroscopic variable capacitor was used (cf. Fig. 13). At low bias voltages, the increase of the internal energy is exponential. At moderate and high voltages (above $15 \mathrm{~V}$ ), the circuit displayed a slowing down and then a saturation of the internal energy of the system. This is because the electrical forces generated by the strongly biased MEMS capacitor interact with the mechanical dynamics of the MEMS 


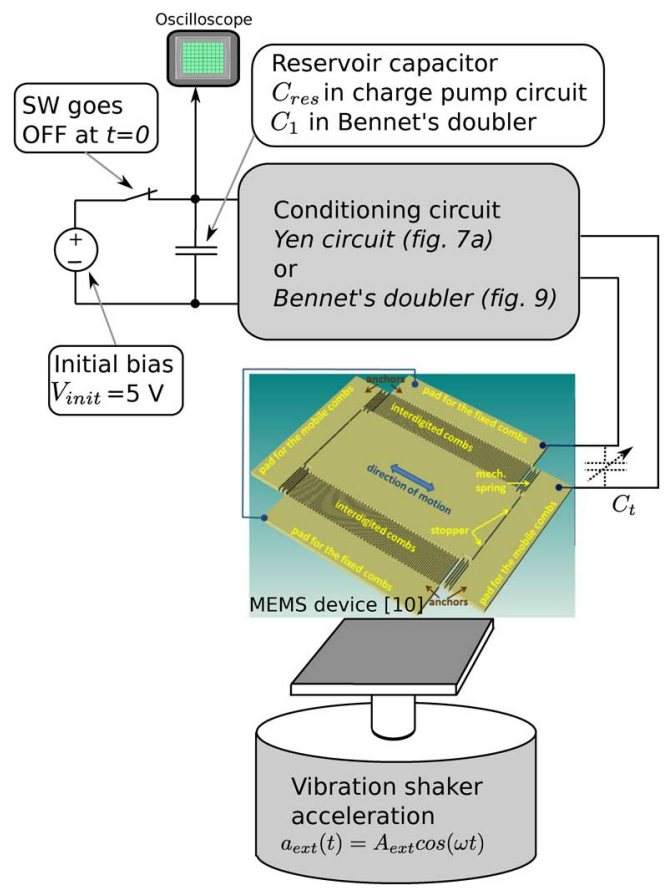

Fig. 12. Diagram describing the experiment highlighting the increase of internal energy of considered conditioning circuits in autonomous mode.

resonator. This results in a modification of the resonance frequency, the pull-in phenomena, etc. Such a behavior cannot be explained in the frame of the purely electrical model used in this article. For that reason, the Part 2 of the article presents a methodology of electromechanical analysis of the system, and provides a formal characterisation of the coupled behavior of the harvester.

Fig. 13(a) presents the evolution of the $V_{\text {res }}$ and $V_{s t}$ voltages in a charge pump obtained in the experimental setup described in [24], as the switch is driven by a fixed periodic time sequence, as in [10]. The autocalibration mechanism was not implemented, but since the parameters of the external vibrations were fixed, this time sequence was optimized so to maximize the output power. The evolution of $V_{s t}$ voltage is locally similar to the theoretical behavior presented in Fig. 8 .

The originality of this experiment is in the use of an integrated MOS switch implemented in a high voltage $0.35 \mu \mathrm{m}$ technology, interfaced with low voltage $(3.3 \mathrm{~V})$ command circuit through a ultra low power static level shifter presented in [16] (cf. discussion at the end of Section III-B).

\section{CONCLUSiOns}

This study, Part 1, presented an original vision unifying the theory of several structurally different conditioning circuits for electrostatic kinetic energy harvesters, by considering the QV cycle as their common and fundamental characteristic. This approach provides a comprehensive methodology for design and optimization, by defining three separate steps: i) selection of the optimal dynamic biasing of the transducer (QV cycle), ii) selection of the architecture implementing it, iii) selection of the optimal electrical mode of this architecture. It was formally shown that the energy yield of the circuits with rectangular QV cycle is determined by the ratio between the extreme voltages $V_{\text {res }}$ and $V_{s t}$ characterizing the cycle geometry. This provides a comprehensive tool for selecting the optimal operation mode as a function of the parameters of the transducer (the ratio $C_{\max } / C_{\min }$ )

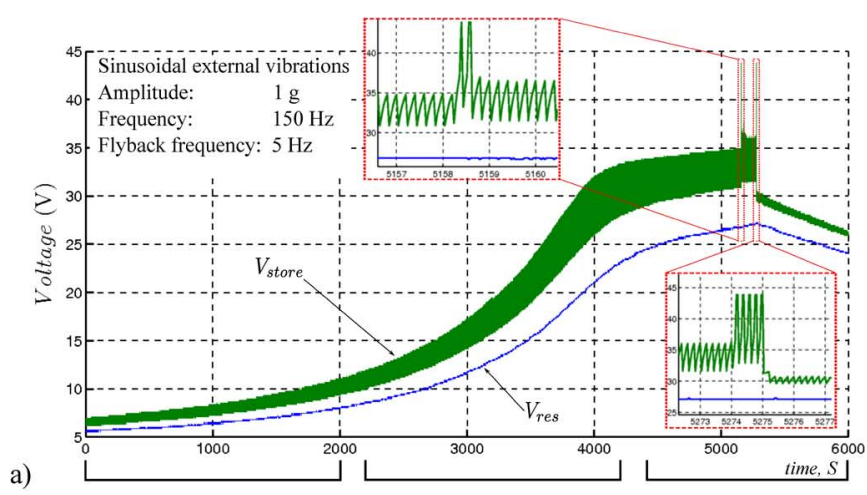

a)

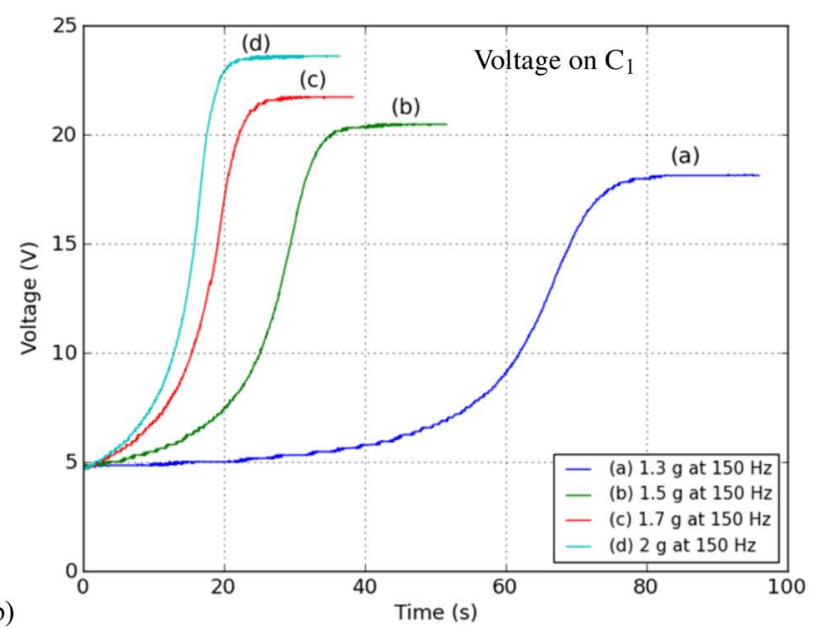

Fig. 13. Experimental results obtained for: (a) charge pump with inductive flyback, measurement setup is described in [24]. (b) Bennet's doubler used with a MEMS device described in [11]. The parameters of the experiment are given in the plots.

or of the electrical limitations imposed by the technological context (maximal supported voltage, maximum available external DC voltage).

The paper considered two families of circuits implementing a rectangular QV cycle: circuits based on the Roundy charge pump, and circuits based on Bennet's doubler. Whereas Bennet's doubler based circuits do not require any external control for steady energy conversion, a charge pump needs to be periodically de-saturated with a flyback circuit, with use of an externally controlled inductive DC-DC converter.

However, the simplicity of implementation of the Bennet's doubler circuit has a cost: an impossibility to adapt its operation to dynamic variation of the ratio $C_{\max } / C_{\min }$. Such a variation is highlighted by both the experiment and the theory; the reason for this variation may be external (variation in the amplitude of the external vibrations) or internal (compressing of the mobile mass amplitude because of the electromechanical coupling). In the both cases, the Roundy charge pump with inductive flyback, when provided with a smart electronics, is able to adapt the voltages $V_{\text {res }}$ and $V_{s t}$ so as to keep an optimal mode of the energy conversion. In the case of the Bennet's doubler, the ratio $V_{s t} / V_{\text {res }}$ is defined by the circuit topology, and can not be dynamically modified. For this reason, the Bennet's doubler is only efficient for a specific range of amplitudes of the mobile mass.

The Bennet's doubler with high multiplication factor is particularly interesting in configurations when the ratio $C_{\max } / C_{\min }$ 
is weak: this is a frequent situation with MEMS capacitive transducers, and this topology has a high potential for practical implementations in microscale harvesters.

The analysis presented in this article was made in the electrical domain: it means that the starting hypothesis is a fixed and known law of the transducer's capacitance variation. The latter requires a knowledge of the amplitude of the mobile mass vibrations, which, in turn, requires a coupled analysis of the system in both mechanical and electrical domains. This is the subject of the Part 2 of the paper.

\section{REFERENCES}

[1] P. D. Mitcheson, E. M. Yeatman, K. G. Rao, A. S. Holmes, and T. C. Green, "Energy harvesting from human and machine motion for wireless electronic devices," Proc. IEEE, vol. 96, no. 9, pp. 1457-1486, 2008.

[2] T. Galchev, E. E. Aktakka, and K. Najafi, "A piezoelectric parametric frequency increased generator for harvesting low-frequency vibrations," J. Microelectromech. Syst., vol. 21, no. 6, pp. 1311-1320, Dec. 2012.

[3] E. Torres and G. Rincón-Mora, "Electrostatic energy-harvesting and battery-charging cmos system prototype," IEEE Trans. Circuits Syst. I, Reg. Papers, vol. 56, no. 9, pp. 1938-1948, 2009.

[4] A. Kempitiya, D.-A. Borca-Tasciuc, and M. M. Hella, "Low-power interface IC for tri-plate electrostatic energy converters," IEEE Trans. Power Electron., vol. 28, no. 2, pp. 609-614, Feb. 2013.

[5] Y. Naruse, N. Matsubara, K. Mabuchi, M. Izumi, and S. Suzuki, "Electrostatic micro power generation from low-frequency vibration such as human motion," J. Micromech. Microeng., vol. 19, p. 094002, 2009.

[6] S. Meninger, J. Mur-Miranda, R. Amirtharajah, A. Chandrakasan, and J. Lang, "Vibration-to-electric energy conversion," IEEE Trans. Very Large Scale Integr. (VLSI) Syst., vol. 9, no. 1, pp. 64-76, 2001.

[7] P. D. Mitcheson, T. Sterken, C. He, M. Kiziroglou, E. Yeatman, and R. Puers, "Electrostatic microgenerators," Meas. Control, vol. 41, no. 4, pp. 114-119, 2008.

[8] S. Roundy, P. Wright, and K. Pister, "Micro-electrostatic vibration-toelectricity converters," in Proc. 2002 ASME Int. Mech. Eng. Congr., Nov. 2002.

[9] E. Blokhina, D. Galayko, P. Basset, and .O Feely, "Steady-state oscillations in resonant electrostatic vibration energy harvesters," IEEE Trans. Circuits Syst. I, Reg. Papers, vol. 60, no. 4, pp. 875-884, Apr. 2013.

[10] B. C. Yen and J. H. Lang, "A variable-capacitance vibration-to-electric energy harvester," IEEE Trans. Circuits Syst. I, Reg. Papers, vol. 53, no. 2, pp. 288-295, 2006

[11] P. Basset, D. Galayko, F. Cottone, R. Guillemet, E. Blokhina, F. Marty, and T. Bourouina, "Electrostatic vibration energy harvester with combined effect of electrical nonlinearities and mechanical impact," $J . M i$ cromech. Microeng., vol. 24, no. 3, p. 035001, 2014.

[12] H. R. Florentino, R. C. S. Freire, A. V. S. Sá, C. Florentino, and D. Galayko, "Electrostatic vibration energy harvester with piezoelectric start-up generator," in Proc. IEEE Int. Symp. Circuits Syst. (ISCAS), 2011, pp. 1343-1346.

[13] A. Nayfeh, Introduction to Perturbation Techniques. New York: Wiley, 1993.

[14] D. Galayko, R. Pizarro, P. Basset, and A. Paracha, "AMS modeling of controlled switch for design optimization of capacitive vibration energy harvester," in Proc. IEEE Int. Workshop Behav. Model. Simul., 2007, pp. 115-120, IEEE.

[15] A. Dudka, D. Galayko, E. Blokhina, and P. Basset, "Smart integrated conditioning electronics for electrostatic vibration energy harvesters," in Proc. IEEE Int. Symp. Circuits Syst. (ISCAS), 2014, pp. 2600-2603.

[16] A. Dudka, D. Galayko, and P. Basset, "Design of controller IC for asynchronous conditioning circuit of an electrostatic vibration energy harvester," in Proc. IEEE Int. Conf. Internet of Things, 2012 Workshop Energy Wireless Sensors.
[17] A. Bennet and R. Kaye, "An account of a doubler of electricity, or a machine by which the least conceivable quantity of positive or negative electricity may be continually doubled, till it becomes perceptible by common electrometers, or visible in sparks," Philos. Trans. Roy. Soc. London, vol. 77, pp. 288-296, 1787.

[18] A. C. M. de Queiroz and M. Domingues, "The doubler of electricity used as battery charger," IEEE Trans. Circuits Syst. II. Exp. Briefs, vol. 58 , no. 12 , pp. 797-801, 2011.

[19] S. Risquez, J. Wei, M. Woytasik, F. Parrain, and E. Lefeuvre, "Self-biased inductor-less interface circuit for electret-free electrostatic energy harvesters," J. Phys., Conf. Series, vol. 557, no. 1, pp. 12 052-12 056, 2014, IOP Publ..

[20] V. Dragunov and V. Dorzhiev, "Electrostatic vibration energy harvester with increased charging current," J. Phys., Conf. Series, vol. 476 , no. 1, p. 012115, 2013, IOP Publ..

[21] V. Dorzhiev, A. Karami, P. Basset, F. Marty, V. Dragunov, and D. Galayko, "Electret-free micromachined silicon electrostatic vibration energy harvester with the bennet's doubler as conditioning circuit," Electron Device Lett., vol. 36, no. 2, pp. 183-135, 2014.

[22] V. Dorzhiev, A. Karami, P. Basset, V. Dragunov, and D. Galayko, "Mems electrostatic vibration energy harvester without switches and inductive elements," J. Phys., Conf. Series, vol. 557, no. 1, pp. 12 126-12 130, 2014, IOP Publ..

[23] D. Galayko, A. Dudka, and P. Basset, "Capacitive kinetic energy harvesting: System-level engineering challenges," in Proc. 21th IEEE Int. Conf. Electron. Circuits Syst., Dec. 2014.

[24] A. Dudka, P. Basset, F. Cottone, E. Blokhina, and D. Galayko, "Wideband electrostatic vibration energy harvester (e-veh) having a low start-up voltage employing a high-voltage integrated interface," $J$. Phys., Conf. Series, vol. 476, no. 1, p. 012127, 2013, IOP Publ..

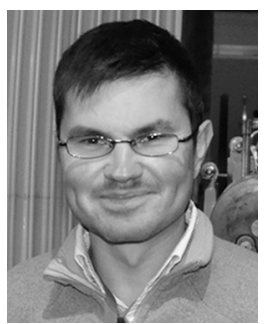

Dimitri Galayko (M'12) graduated from Odessa State Polytechnich University, Ukraine, in 1998; he received his master degree from Institut of Applied Sciences of Lyon, INSA-LYON, France, in 1999. He created his Ph.D. thesis in the Institute of Microelectronics and Nanotechnologies (IEMN), Lille, France, and received the Ph.D. degree from the University Lille-I in 2002. The topic of his Ph.D. dissertation was the design of microelectromechanical silicon filters and resonators for radiocommunications. Since 2005 he is an Associate Professor at University of Paris VI (UPMC, Sorbonne Universités), France, in the LIP6 laboratory. His research interests include study, modeling, and design of nonlinear integrated circuits for sensor interface and for mixed-signal applications.

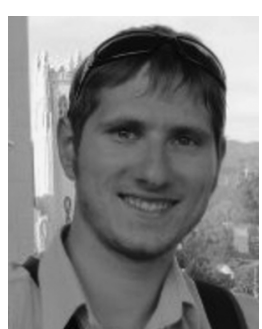

Andrii Dudka received the M.S. degree in computer science and electronics from Odessa National Polytechnic University, Ukraine, in 2008, and the Ph.D degree in electrical engineering from the Pierre and Marie Curie University, lab. LIP6, France, in 2014. $\mathrm{He}$ is currently a Postdoctoral Researcher at University of Bordeaux, lab. IMS, France. His research interests are in the areas energy harvesting from vibrations, micropower generation and conversion using MEMS devices, analog electronics, and ASICs.

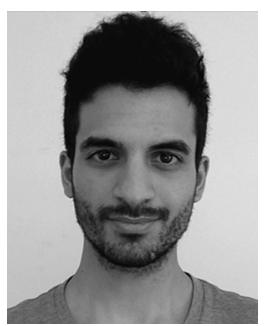

Armine Karami was born in Paris, France, in 1991 He received the M.S degree in electrical engineering from Université Pierre et Marie Curie-Paris 6 (UPMC), France, in 2014. He is currently pursuing the Ph.D. degree at Université Pierre et Marie Curie (UPMC) - Paris 6, at the Laboratoire d'Informatique de Paris 6. His Ph.D. research is focused on the theory, conception and analysis of conditioning circuits for vibration energy harvesting. 


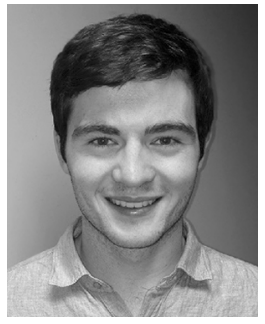

Eoghan O'Riordan received the B.E. degree in electrical engineering from University College Dublin (UCD), Ireland, in 2012. He is currently working toward the Ph.D. degree at UCD. His research interests include nonlinear dynamics, and vibration energy harvesting.

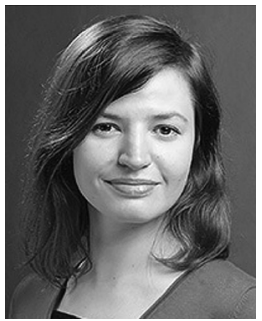

Elena Blokhina (S'05-M'06-SM'13) received the M.Sc. and Ph.D. degrees in physics from Saratov State University, Saratov, Russia, in 2002 and 2005 , respectively. From 2005 to 2007, she was a Research Scientist at Saratov State University, where she worked in the area of analysis and modeling of microwave electron devices. Since 2007, she has been with University College Dublin, Dublin, Ireland, where she currently is a lecturer and the research manager of the Circuits and Systems Research Group. Her research interests include nonlinear dynamics and oscillation theory and their application to the analysis of MEMS and energy harvesters. Dr. Blokhina is a member of the IEEE Technical Committee on Nonlinear Circuits and Systems. She has been elected to serve in the Board of Governors of the IEEE Circuits and Systems society for the term 2013-2015. She has served as a reviewer and review committee member for a number of international journals and conferences on circuits and systems, nonlinear dynamics and numerical modelling. In 2013 and 2014, she has served as the track co-chair for Nonlinear Circuits and Systems, Neural Networks at the IEEE International Conference on Electronics, Circuits and Systems and the track chair for Nonlinear Circuits and Systems as the IEEE International Symposium of Circuits and Systems.

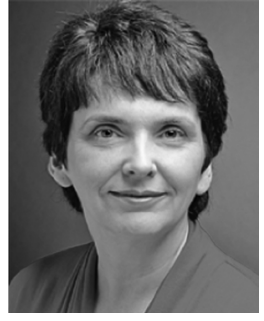

Orla Feely (S'85-M'86-SM'00-F'09) received the B.E. degree in electronic engineering from University College Dublin (UCD), Ireland, in 1986, and the M.S. and Ph.D. degrees in electrical engineering from the University of California, Berkeley, CA, USA, in 1990 and 1992, respectively. She joined UCD in 1992 and is currently Vice-President for Research, Innovation and Impact. Her research interests lie in the area of nonlinear dynamics of electronic circuits. Prof. Feely is a Fellow of Engineers Ireland and the Irish Academy of Engineering. She received the Best Paper Awards of the International Journal of Circuit Theory and Applications, 2007, and the European Conference on Circuit Theory and Design, 1997. Her Ph.D. thesis won the D. J. Sakrison Memorial Prize for outstanding and innovative research, awarded annually by the Department of Electrical Engineering and Computer Science, U. C. Berkeley. She serves/has served on the Editorial Boards of the IEEE TRANSACTIONS ON CIRCUITS AND SYSTEMS and the International Journal of Circuit Theory and Applications, and as Chair of the IEEE Technical Committee on Nonlinear Circuits and Systems. Prof. Feely is Chair of the Irish Research Council.

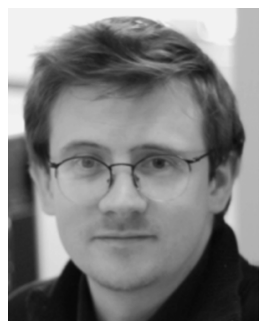

Philippe Basset received the engineering diploma in electronic from ISEN Lille, France, in 1997, and the M.Sc and Ph.D degrees from IEMN University of Lille, France, in 1999 and 2003 respectively. In 2004 he was a Postdoc at CMU, Pittsburgh, PA, USA. In 2005 he joined ESIEE Paris at the Universit Paris-Est, France, where he is currently an Associate Professor. His research interests include micro/nano-structuration of silicon and micropower sources for autonomous MEMS. He led several projects on energy harvesting using micro- and nano-technologies and he serves in the TPC of the PowerMEMS conference since 2013. A member of the ESYCOM laboratory, he is currently leading the Sensors and Measuring MEMS group. 